\title{
Metodología de investigación en Salud Laboral
}

\section{Research methodology in occupational health}

\author{
Juan Antonio Mirón Canelo \\ Departamento de Medicina Preventiva y Salud Pública. \\ Facultad de Medicina. Universidad de Salamanca. Salamanca. España.
}

\section{Montserrat Alonso Sardón}

Departamento de Medicina Preventiva y Salud Pública.

Facultad de Medicina. Universidad de Salamanca. Salamanca. España.

\author{
Helena Iglesias de Sena \\ Departamento de Medicina Preventiva y Salud Pública. \\ Facultad de Medicina. Universidad de Salamanca. Salamanca. España.
}

\section{Correspondencia:}

José Antonio Mirón Canelo

Departamento de Medicina Preventiva y Salud Pública

Facultad de Medicina

Universidad de Salamanca

Campus de Miguel de Unamuno s/n.

37007 - Salamanca. España.

Teléfono: +34923294500 (Extensiones 1817)

Coreo electrónico: miroxx@usal.es

\section{ResUmen}

El objetivo de este artículo es dar a conocer los principales diseños utilizados en investigación aplicada, clínica o de Salud Laboral. Se analiza su estructura básica y el proceso llevado a cabo en un proyecto de investigación.

Se describen los métodos de investigación, sus utilidades, su interpretación y su aplicación práctica en relación con la obtención de información fiable y válida que permita una lectura crítica de la comunicación científica, generalmente artículos científicos. La calidad, validez y fiabilidad de la información generada por un trabajo de investigación determinado dependen del diseño y metodología y del proceso llevado a cabo por el equipo de investigación.

Su utilidad fundamental es generar evidencias científicas necesarias e imprescindibles para la toma de decisiones, adecuadas y pertinentes, en relación con la prevención, atención y rehabilitación de las personas que padecen enfermedades relacionadas con el trabajo, enfermedades profesionales o accidentes laborales. También, constituye una herramienta imprescindible en el Desarrollo Profesional Continuo, siendo el elemento determinante del autoaprendizaje a través de la lectura crítica de los artículos científicos.

Med Segur Trab (Internet) 2010; 56 (221): 347-365

Palabras clave: Investigación aplicada o clinica, diseños, Salud Laboral.

Abstract

The aim of this article is to present the main designs used in applied and clinical research or occupational health. We analyze the basic structure and the process conducted in a research project.

The article describes the research methods, usefulness, interpretation and practical application in relation with obtaining reliable and valid information for a critical reading of scientific communication, 
scientific articles generally. The quality, validity and reliability of the information generated by a research depend on the design and methodology and the process carried out by the research team.

The fundamental usefulness of this article is to generate scientific evidence, necessary and essential to make appropriate decisions in relation with the prevention, care and rehabilitation of people with workrelated diseases, occupational diseases or accidents. Also, it is essential in Continuing Professional Development, remain the key element of self-learning through critical reading of scientific articles.

Med Segur Trab (Internet) 2010; 56 (221): 347-365

Key words: Applied or clinical research, design, occupational bealth. 


\section{IMPORTANCIA}

En la Sociedad actual del conocimiento y la información es preciso disponer de herramientas y procedimientos de investigación que permitan conocer las evidencias científicas sobre problemas de Salud de los trabajadores para tomar decisiones en relación con la prevención, higiene y seguridad ocupacional y rehabilitación e inserción laboral.

La Epidemiología tiene entre sus aplicaciones y objetivos conocer la historia natural de las enfermedades y los problemas de salud. Para cumplir este fin se vale de distintos diseños epidemiológicos, que correctamente utilizados permiten obtener observaciones, resultados e información que una vez validada permite tomar decisiones en los distintos ámbitos profesionales para contribuir a la mejora de la atención y del bienestar de los pacientes, usuarios y/o trabajadores. También, antes de la toma de decisiones, los profesionales deben evaluar la validez de las observaciones que se realizan en la comunicación científica, ya sea un artículo científico, un informe profesional o una auditoría externa.

El objetivo de este artículo es dar a conocer los principales diseños y procedimientos utilizados en investigación aplicada, clínica o de Salud Pública para tratar de conocer la validez y/o rigor científico de la información generada a través de un trabajo de investigación que ha sido publicado e indexado en una fuente de datos.

Se describen los métodos de investigación, sus utilidades, su interpretación y su aplicación práctica en relación con la búsqueda de datos e información fiable y válida que permita una lectura crítica de la comunicación científica, generalmente artículos científicos, desde el punto de vista de la calidad de la información generada por el tipo de estudio realizado - diseño y metodología- y por el proceso llevado a cabo por el equipo de investigación.

Su utilidad fundamental es generar evidencias científicas necesarias e imprescindibles para la toma de decisiones, adecuadas y pertinentes, en relación con la prevención, atención y rehabilitación de las personas que padecen enfermedades relacionadas con el trabajo, enfermedades profesionales o accidentes laborales. También, constituye una herramienta imprescindible en el Desarrollo Profesional Continuo, siendo el elemento determinante del autoaprendizaje a través de la lectura crítica de los artículos científicos.

\section{INVESTIGACIÓN: CONCEPTOS PREVIOS Y/O BÁSICOS}

La práctica asistencial y profesional es un proceso permanente de toma de decisiones sobre el estado de salud de un individuo, sus problemas y necesidades, que se basa en la información obtenida de las investigaciones realizadas. La dinámica asistencial requiere de datos e información, que referidos a un paciente, a un trabajador o a una situación, permiten realizar un diagnóstico e instaurar unas medidas específicas. Estos datos cuantitativos constituyen la parte objetiva y científica de la práctica de la Medicina y de la Salud Laboral, la parte subjetiva forma parte del arte de aplicar a cada paciente, usuario o trabajador lo adecuado y correcto de acuerdo con las evidencias científicas desarrolladas por la investigación.

Investigar significa "hacer diligencias para descubrir una cosa”, e investigación es la "acción de investigar". Se considera como tal cualquier proceso o actividad que desarrolla un proyecto de forma sistemática y organizada y que tenga por finalidad ampliar e innovar los conocimientos que se tienen en un momento dado sobre un determinado problema de salud y/o enfermedad. El fin fundamental de la investigación, en general, es analizar y interpretar de manera objetiva la realidad, y en caso específico de la Salud, tratar de describir, analizar y explicar los problemas de Salud relacionados con un individuo, grupos poblacionales o comunidad. 
La investigación se clasifica, según el objetivo esencial, en dos tipos:

- Básica: aquella que se realiza con el objetivo de aumentar el acervo de conocimientos científicos (mediante esta investigación es como se producen los avances de las Ciencias). Son por tanto, aquellos conocimientos que suponen la base de las evidencias científicas (MBE). Ej.: Estudios genéticos sobre las enfermedades, tratar de desarrollar y elaborar una vacuna frente a una enfermedad infecciosa profesional. Tratar de explicar las bases moleculares de los signos y los síntomas, etc.

- Aplicada o Clínica o Salud Pública: es aquella que no pretende aportar nuevos conocimientos científicos, sino facilitar la aplicación práctica de los conocimientos ya existentes (este tipo de investigación tiene gran interés desde el punto de vista de la Salud Pública). Ej.: Realizar un estudio epidemiológico para averiguar la frecuencia de una enfermedad profesional, estudiar los factores de riesgo asociados a la enfermedad laboral, valorar mediante un protocolo la mejora de la Calidad de Vida de los trabajadores después de un programa de intervención, etc.

Recientemente se viene introduciendo esta distinción en las distintas convocatorias de ayudas destinadas a financiar proyectos de investigación y que realizan en nuestro país distintos organismos e instituciones estatales y autonómicas (FIS, Ministerios y Comunidades Autónomas). Las dos son compatibles y necesarias, la primera aumenta el conocimiento y la segunda valorar la aplicación y efectividad de los mismos a la realidad social.

La palabra método significa "camino hacia". Sería el procedimiento para conseguir los objetivos propuestos en la investigación. El método científico se refiere, por tanto, al procedimiento ordenado y sistemático que se utiliza para conseguir los objetivos propuestos y, trata de contestar a las preguntas e interrogantes que se plantean en un proyecto de investigación. Proceder con método significa seguir el camino más fácil y racional que lleva a un objetivo.

El objetivo es el eje en torno al cual se desarrolla un diseño del estudio y es la base para decidir los criterios de selección de los trabajadores o pacientes y las variables epidemiológicas - independientes y dependientes - que van a ser medidas y evaluadas en la investigación. A partir de un objetivo general que se pretende estudiar, se realizan matizaciones y se establecen, generalmente, una serie de objetivos específicos, más concretos.

El modo de actuar de la razón humana es básicamente de dos formas:

- Desde observaciones particulares a la proposición general o universal: método inductivo. Utilizado por las Ciencias Fácticas como las Ciencias de la Salud (Medicina, Biología, Química, etc.). Forma habitual de proceder en la mayoría de los estudios e investigaciones.

- Desde proposiciones generales a las premisas particulares: método deductivo. Utilizada por las Ciencias Lógicas como la Lógica y la Matemática.

El método epidemiológico es la aplicación del método científico experimental al estudio de los problemas de Salud. El primer profesional sanitario que utilizó el método de manera ordenada por fases fue John Snow. Este médico rural inglés aplicó el método al estudio de las epidemias de cólera que ocurrieron en Londres en 1853 y 1854. Debido a ello, se le considera el padre del método epidemiológico.

Epidemiología: es básicamente una herramienta de trabajo y estudio para las Ciencias de la Salud y los profesionales sanitarios. Para conseguir objetivos, tales como: frecuencia de siniestralidad laboral, accidentes de trabajo, causas y factores de riesgo de enfermedades ocupacionales y/o profesionales. 
Etapas: 1. Observación del problema de Salud Laboral.

2. Recogida y tabulación de los datos e información.

3. Elaboración y formulación de la hipótesis.

4. Experimentación (verificación/rechazo) de la hipótesis.

5. Emisión del informe o formulación de ley.

Para las primeras dos etapas, se requiere un buen sistema de información sobre la distribución y frecuencia de una enfermedad común en una población o un ámbito laboral, en un área y en un tiempo definido. Este conocimiento puede ser directo, por observación personal como ocurre en la práctica asistencial o laboral, o puede ser indirecto cuando surge de la recogida de datos e información de los diferentes registros, que conforman el Sistema de Información Sanitaria (SIS), y también de la revisión bibliográfica obtenida de las fuentes de datos. Los datos e información disponibles en los distintos registros de mortalidad y morbilidad del SIS español se pueden y se deben utilizar por parte de los profesionales sanitarios para valorar el contexto epidemiológico y social. Los datos obtenidos de los mismos son datos secundarios, dado que no proceden de investigaciones sino de la práctica asistencial habitual. Estos datos proceden de múltiples observaciones y dan lugar a información poblacional. Mientras que los datos obtenidos por un médico de empresa o de Salud Laboral dan lugar a información para elaborar un informe o estudio que sólo se refiere a su ámbito laboral y a trabajadores que pueden o no ser representativos.

Con respecto a la revisión bibliográfica que debe hacerse como punto de partida de la investigación, hemos de decir, que debe basarse en una búsqueda bibliográfica sistemática, ordenada y específica en relación con el objetivo del estudio. La forma tradicional es la consulta de las distintas revistas científicas de donde se obtiene la información por las diferentes investigaciones publicadas por otros autores. Son, por tanto, datos primarios provenientes de trabajos de investigación publicados e indexados.

La revisión de la bibliografía constituye una herramienta imprescindible en el sector sanitario dado que facilita la puesta al día en base a las investigaciones existentes y puede realizarse de dos formas. En primer lugar, con una finalidad narrativa y sin materiales y método predefinidos (Revisión Clásica). En segundo lugar, a través de la búsqueda sistemática de la evidencia científica disponible utilizando una metodología predefinida y, en su caso, integrando resultados mediante metaanálisis. Esta última revisión, de mayor calidad, sirve para facilitar la toma de decisiones profesionales de manera competente.

En el caso concreto de trabajos relacionados con Salud Laboral y Medicina del Trabajo, a la hora de establecer la estrategia de búsqueda, resulta de gran utilidad recurrir a filtros metodológicos especialmente diseñados y validados para esta disciplina científica.

Las principales bases de datos en las Ciencias Biomédicas son las siguientes:

- Index Medicus. Editado por la National Library of Medicine (NLM) de Estados Unidos, recoge anualmente información sobre unas 4.000 revistas médicas de todo el mundo. Se publica desde 1966. Su información se recoge en la base de datos MEDLINE. Es la base de datos más utilizada, entre otras razones, por ser gratuita.

- Excerpta Medica. Editado por la Fundación Internacional Excerpta Medica de Ámsterdam (Holanda). Se publica por series temáticas desde 1980. Tiene carácter selectivo y analítico. Incluye unas 4.500 revistas, fundamentalmente europeas. También incluye libros. Su información se recoge en la base datos EMBASE.

- Current Contens y Science Citation Index. Editado por el Institute for Scientific Information (ISI) de Filadelfia (Estados Unidos). Tiene información de unas 2.000 revistas que se recoge en la base de datos SCISEARCH. 
- Indice Bibliográfico Español de Ciencias de la Salud. Editado por el Instituto de Salud Carlos III, contiene referencias de artículos de revistas científicosanitarias editadas en España e incluye contenidos de las diferentes ramas de las ciencias de la salud tales como Medicina (incluyendo Salud Pública, Epidemiología y Administración Sanitaria), Farmacia, Veterinaria, Psicología, Odontología y Enfermería. Su versión informatizada se denomina IBECS.

- Índice Médico Español. Editado por el Instituto Valenciano de Investigación. Recoge información sobre unas 200 revistas españolas del ámbito de la Medicina y otras ciencias afines. Su versión informatizada se denomina IME.

- Cochrane Library. Es una base de datos orientada a la tomas de decisiones clínicas, principalmente diagnósticas y terapéuticas. Se basa en revisiones sistemáticas y metaanálisis.

\section{HIPÓTESIS Y/O OBJETIVOS DEL ESTUDIO O INVESTIGACIÓN}

Una vez revisada la bibliografía y habiendo obtenido una idea global sobre lo que se conoce en un momento dado, el siguiente paso que debe dar el profesional a la hora de investigar, es seleccionar una pregunta relevante, suficientemente precisa y acotada, no respondida anteriormente (o al menos no en su ámbito laboral o profesional), factible de ser respondida y, por último, pertinente.

Se trata de la tercera etapa del método epidemiológico. La idea particular o el problema se expresa en forma de hipótesis y/o por medio de objetivos. En general, la generación de hipótesis de trabajo, o simplemente la apertura de un nuevo ámbito de estudio, es un asunto creativo e innovador. Posteriormente, un proceso lógico irá perfilando la idea o la hipótesis sobre la base de una revisión — generalmente bibliográficade lo conocido hasta el momento sobre el tema, enfermedad o problema.

La hipótesis sintetiza la pregunta científica a la que se pretende dar respuesta en la investigación y permite enjuiciar a los evaluadores en relación con la originalidad, factibilidad y relevancia científica y sociosanitaria del proyecto.

Posteriormente, viene la verificación o comprobación de la hipótesis, que se hace por medio de estudios epidemiológicos (descriptivos, analíticos o experimentales). Entramos en la cuarta etapa del método. Para el proceso de prueba o verificación de la hipótesis se deben tener en cuenta los aspectos de:

Validez de la información, es decir, grado en el que un estudio mide el objetivo que se propone o aquello para lo que ha sido diseñado (procedimiento destinado a evitar errores y/o errores sistemáticos o sesgos).

- Validez interna: grado en que los resultados de la investigación responden a las preguntas que se hace el equipo investigador y a los objetivos planteados en el estudio. Se ve afectada por los errores sistemáticos y por los factores de confusión.

- Validez externa: grado en que las conclusiones pueden generalizarse a la población diana o de referencia. Se ve afectada por la selección de la muestra y por los errores aleatorios y sesgos.

- Precisión, fiabilidad o reproducción del estudio: posibilidad de reproducción de un estudio bajo las mismas condiciones con resultados similares (procedimiento destinado a controlar los errores de muestreo).

- Exactitud: mecanismo encargado de evitar tanto los sesgos como los errores de muestreo (combinación de validez y precisión).

El objetivo es aquello que se dirige hacia una meta concreta y precisa. Los objetivos consisten en una guía para el trabajo, dependiendo de la hipótesis planteada, de acuerdo con el problema, la idea central o con los propósitos que se establecen. 
Tienen por finalidad:

- Determinar el trabajo que debe hacerse.

- Identificar las partes principales del problema, así como la relación que existe entre ellas.

- Seleccionar el procedimiento o el método adecuado para la realización del trabajo.

- Programar el trabajo en términos de unidades de tiempo y procedimiento.

Los objetivos, por dirigir la idea principal de la investigación hacia un fin, permiten un mejor aprovechamiento del trabajo requerido y una adecuada coordinación de esfuerzos para alcanzar los resultados últimos.

Un trabajo de investigación en Salud puede no tener hipótesis, pero siempre tendrá una idea central o directriz que se encaminará a través de los objetivos. Una revisión exhaustiva de los trabajos existentes permitirá conocer cuáles son los posibles reductos o lagunas que quedan por investigar, establecer cuáles son nuestras hipótesis de trabajo y cuáles los objetivos principales (generales) y secundarios (específicos) del estudio.

\section{RELEVANCIA DEL PROYECTO}

Este apartado trata de describir y explicar la realidad del problema de salud o enfermedad y su importancia sanitaria y social (impacto). La necesidad de justificar la relevancia del estudio viene determinada por la necesidad de conocimiento de la comunidad científica y de la Sociedad. A este respecto, deben discutirse y valorarse las razones que llevan al equipo investigador a plantear la investigación: la magnitud del problema (valorada a través de indicadores de morbilidad y mortalidad que se obtienen de los registros que forman parte del Sistema de Información Sanitaria), los conocimientos actuales sobre el tema (valorados mediante una revisión bibliográfica) y la oportunidad en el tiempo e impacto sanitario y social del mismo.

En general, no es suficiente que un proyecto de investigación tenga como última finalidad el cubrir una laguna de conocimiento, es necesario que, además, los resultados se puedan aplicar en la práctica habitual y tengan impacto sanitario y/o social.

En cualquiera de los casos, es preciso tomar en consideración cuáles son las líneas prioritarias de investigación en cada momento, bien las que parten de las Instituciones y Organismos que financian las investigaciones (FIS, I+D+I, Ministerio de Trabajo, Comunidades Autónomas, otros), bien las que emanan de las situaciones concretas de los estados de Salud o de los servicios sanitarios (Cáncer ocupacional, seguridad e higiene ante nuevos factores y situaciones de riesgo laboral, enfermedades profesionales emergentes o nuevas, calidad de vida relacionada con la Salud, etc.). Estas líneas serán además las que orienten la pertinencia o no de un determinado proyecto.

En función de este proceso de estudio previo, antecedentes y bibliografía, se justifica la investigación y se procede a fijar los objetivos generales y específicos de la misma que deben reflejarse en la introducción.

\section{METODOLOGÍA EN INVESTIGACIÓN: DISEÑO}

Uno de los aspectos más importantes al proponer un proyecto de investigación es la elección del diseño de investigación. El objetivo de éste es obtener información sobre un problema de Salud determinado al que el equipo de investigación intenta dar una respuesta objetiva y válida. No se trata de una mera recogida de datos, sino de un planteamiento que se deriva de los objetivos que se persiguen y de la estrategia y capacidad necesaria para cumplirlos. 
El diseño epidemiológico de un estudio se define como el conjunto de procedimientos, métodos y técnicas mediante las cuales un equipo de investigadores selecciona los trabajadores, recopila información y analiza los resultados del estudio de investigación. Permite, según el tipo de investigación, mejorar los conocimientos existentes y obtener respuestas que faciliten la toma de decisiones en relación con la seguridad e higiene y la Salud Laboral.

El abanico de posibles diseños es muy amplio y la elección del diseño epidemiológico adecuado se determinará en función de dos parámetros básicamente como son la validez y la viabilidad del estudio. En primer lugar, se trata de evitar que un trabajo o investigación quede invalidado por la falta de un diseño correcto, que ningún estadístico o interpretación sesgada pueda salvar. En segundo lugar, se trata de que sea posible realizarlo en las circunstancias habituales de trabajo. El balance entre estos dos elementos marcará el proceso de planificación del proyecto de investigación que se va a realizar.

El planteamiento metodológico comprenderá básicamente: la selección del tipo de estudio, la lista de variables a valorar, incluyendo su nivel de medición, el tipo de sujetos de observación, la fuente de los sujetos a incluir, el tamaño de muestra adecuado y el procedimiento de intervención o de observación para la toma de los datos.

\section{Tipos de estudios}

La selección del tipo de estudio depende de la hipótesis y de los objetivos. Los principales tipos de diseños epidemiológicos se clasifican:

\section{Según la orientación del estudio en el tiempo}

- Estudios Longitudinales: estudian los fenómenos a lo largo del tiempo, permiten estudiar a los sujetos en varios momentos a lo largo de un período de tiempo. Aspecto que facilita la determinación de la secuencia de los acontecimientos (secuencia temporal y frecuencia). Obtienen una película de lo que pasa.

- Estudios Transversales: permiten observar un proceso sólo en un momento dado, valorando exposición (FR) y efecto (E) o desenlace en un mismo momento (sólo permiten aventurar la hipótesis sobre qué factor precede a otro). Obtienen una foto de situación.

\section{Según la orientación del proceso}

- Estudios Retrospectivos:

$$
\mathrm{E} \Rightarrow \mathrm{FR}
$$

- Estudios Prospectivos: $\quad$ FR $\Rightarrow \mathrm{E}$

Términos que hacen referencia a la relación temporal existente entre el inicio del estudio por el equipo investigador y la aparición del desenlace o efecto. Los prospectivos son los más adecuados dado que permiten obtener incidencias, una mejor planificación y control del estudio lo que mejora la validez de los resultados.

\section{Según los objetivos básicos de la investigación}

- Estudios Descriptivos: describen las características generales de la distribución del problema de Salud laboral en relación con las variables de persona, lugar y tiempo, lo que permite generar hipótesis y servir a la planificación sanitaria, es decir, a la toma de decisiones en el ámbito laboral.

- Estudios Analíticos: se plantean para contrastar hipótesis entre dos o más grupos, con el objetivo de que la comparación sirva para comprobar la hipótesis. Los analíticos prospectivos, denominados cohortes, permiten mediante el seguimiento valorar y comparar un grupo de trabajadores expuestos a un factor de riesgo ocupacional frente a otro grupo no expuesto. 


\section{Según exista o no manipulación en el estudio}

- Estudios Observacionales: el investigador mide pero no interviene, es decir, no manipula las variables epidemiológicas, solo las observa, las mide mediante indicadores de frecuencia y las interpreta.

- Estudios Experimentales: el equipo investigador puede manipular a su voluntad la relación causa-efecto, existe asignación controlada - manipulación- del factor sometido a estudio.

Para tener una visión global del conjunto de diseños se puede observar la figura 1, que no siendo exhaustiva, ofrece una clasificación sencilla y fácil de comprender.

Figura 1. Tipo de diseños

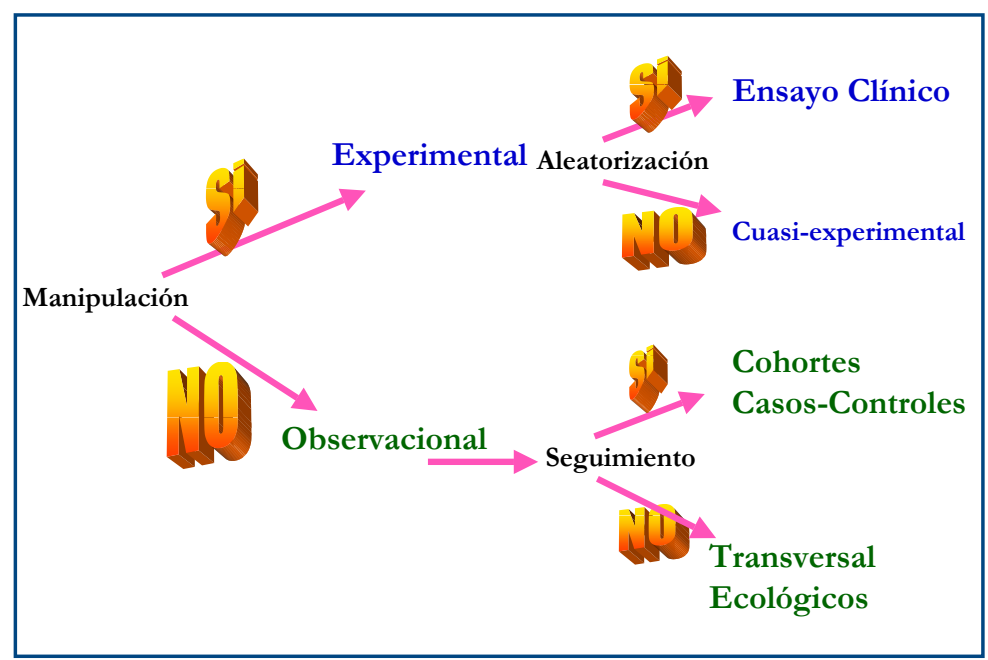

\section{ESTUDIOS OBSERVACIONALES}

Cuando simplemente el equipo investigador se limita a observar lo que sucede y no se tiene ningún control. Dentro de ellos, un segundo factor nos hará optar por uno u otro diseño: el conocimiento previo sobre el tema a estudiar.

\section{Estudios Observacionales Descriptivos}

Cuando no se conozca nada, o casi nada, sobre el tema a investigar. Nos ofrecen una primera aproximación a la perspectiva y distribución de un problema de Salud mediante la simple observación del mismo en un determinado momento y contexto. El objetivo es describir las características y frecuencia de un problema de salud en función de las variables de persona (edad, sexo, ocupación, nivel de instrucción, raza, ingresos,...), de lugar (ámbito laboral para determinar el Mapa de riesgos de un Área de Salud ...), y de tiempo (variaciones cíclicas interanuales, polianuales, estacionales, mensuales, semanales ...). Su principal utilidad: Planificación Sanitaria (toma decisiones, aplicando medidas, actividades o programas) y generar hipótesis que son la base de los estudios observacionales analíticos posteriores. Estos estudios se clasifican en:

\section{Individuales}

Informe de un caso / Serie de casos: es una aproximación descriptiva que se basa en la experiencia laboral/ocupacional de un trabajador o grupo de trabajadores con un diagnóstico similar. Este tipo de estudios no sirven para probar hipótesis, dado que representan la experiencia de un solo trabajador o un pequeño número sin existir un grupo control.

Estudios de prevalencia, de corte o transversales: estudian la situación de un problema de Salud Laboral en un momento determinado. Es posible identificar a cada uno de los trabajadores de los ámbitos ocupacionales que estudiamos. Proporcionan datos útiles desde el punto de vista de la Planificación y Gestión Sanitaria (identificando 
problemas, necesidades y grupos de mayor riesgo o vulnerabilidad) y son útiles para evaluar intervenciones sobre la población laboral. Al comparar con otros grupos o poblaciones generales o de referencia en la distribución de los problemas o de los factores de riesgo, permiten hallar diferencias significativas, no se deben al azar, que sirven para generar hipótesis útiles en el diseño posterior de estudios analíticos. Sus ventajas son: fáciles de realizar, repetibles y baratos.

\section{Poblacionales}

Estudios Ecológicos: la unidad de estudio son sectores ocupacionales o poblaciones y no individuos en sí (no es posible identificar a cada uno de los trabajadores de los grupos de población que estudiamos). Requieren la existencia de datos agregados, ambientales o globales. Permiten relacionar, a nivel grupal poblacional, dos variables determinadas en distintos ámbitos geográficos, o temporales. Se apoyan en fuentes de datos e información que han sido previamente publicados. La progresiva informatización de los datos, su accesibilidad a través de la red de internet y la confidencialidad de los datos que impide el uso de la información de manera individualizada, está posibilitando la proliferación de este tipo de estudios. Son utilizados por su potencial para generar bipótesis, aunque sin llegar a poder establecer asociación o causalidad entre las variables a estudio. Su mayor sesgo es realizar deducciones individuales a partir de los datos agregados. Sesgo que se denomina falacia ecológica. De datos agregados, grupales y/o poblacionales se extrapola y se llega a conclusiones individuales. Ejemplo: la alta incidencia y asociación de tuberculosis (TB) en mineros, no nos permite decir que un determinado trabajador de la minería tiene un alto riesgo o probabilidad de padecer TB y/o silicosis. Hay trabajadores que trabajan en la minería; pero no necesariamente bajan a la mina y no todos tienen el mismo grado de exposición laboral. Las ventajas de este tipo de estudio son la rapidez y el bajo coste. Dentro de los estudios ecológicos existen varios tipos:

- Descriptivos o de mapas: buscan la detección de patrones geográficos.

- Series temporales: comportamiento de los problemas de Salud a lo largo del tiempo.

- Estudios de correlación ecológica: relación entre efectos y nivel de exposición en diferentes poblaciones.

\section{Estudios Observacionales Analíticos}

Cuando ya existen conocimientos previos sobre la distribución de una determinada enfermedad profesional o efecto ocupacional y queremos explicar el porqué de ese comportamiento (cuál es la causa -causalidad-, qué factores de riesgo están relacionados - asociación-, en qué medida, o qué cantidad del efecto es atribuible a un factor determinado -riesgo-). Se debe hacer una aproximación de tipo observacional (no experimental) que representa la posibilidad de verificación de la hipótesis. Pueden ser a su vez de dos tipos:

Estudios de Cohortes o de seguimiento: se seleccionan dos grupos de individuos que difieren en cuanto a su exposición o no al factor de riesgo (FR) que queremos estudiar; se siguen en el transcurso del tiempo — carácter longitudinal del diseño_, y son comparados respecto a la incidencia del efecto (E) - carácter prospectivo del diseño $(\mathrm{FR} \Rightarrow \mathrm{E})$ - Es decir, un grupo de trabajadores que llamamos cohorte (tienen en común alguna característica, trabajan en el mismo sector laboral) ha estado expuesta a un factor de riesgo (FR) determinado: cohorte expuesta. Se procede a comparar la frecuencia de aparición del efecto (E) de interés en este grupo con respecto a otro de características similares, pero que no ha esta sometido a dicha exposición: coborte no expuesta. Son estudios longitudinales (los trabajadores están sometidos a varias observaciones), prospectivos (hacia delante y permiten el cálculo de incidencias y RR) y estudian la relación causal de la causa al efecto o enfermedad. En la figura 2 se puede observar la estructura básica de este tipo de estudios. 
Figura 2. Estructura básica de un diseño de Cohortes

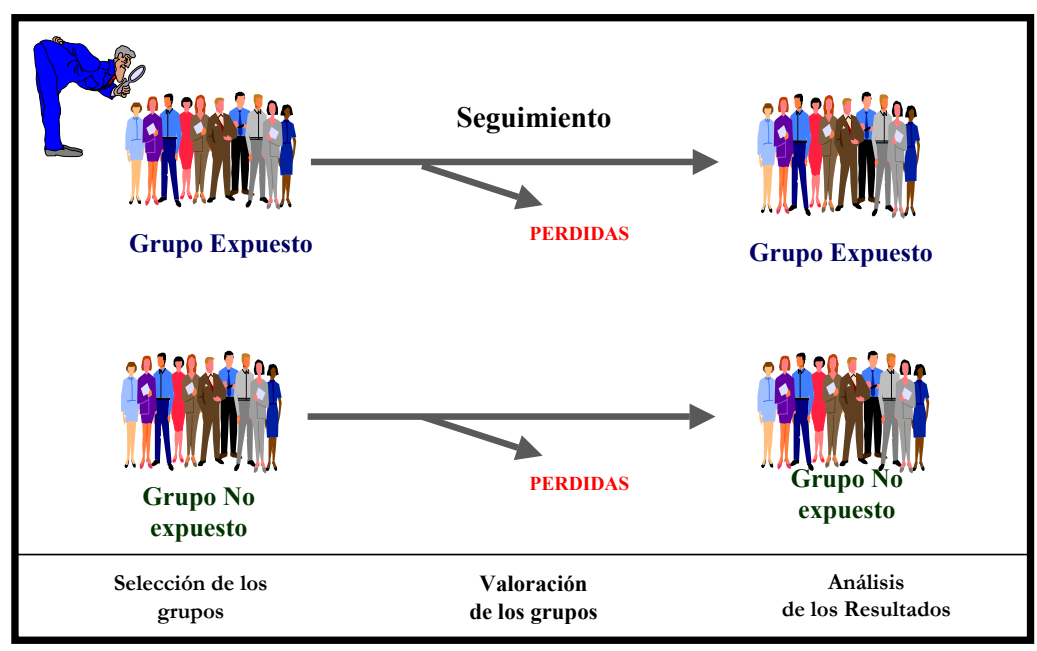

Ventajas: cálculo de incidencias (RR), tienen secuencia temporal — de la causa al efecto-, permiten una buena planificación de del estudio y control de los sesgos y analizar varias exposiciones o riesgos ocupacionales a la vez.

Inconvenientes: tiempo necesario de seguimiento entre la exposición y el efecto ocupacional, las pérdidas de trabajadores por diferentes motivos, coste elevado, por lo que no son adecuados ni efectivos para estudiar enfermedades laborales y/o profesionales raras ni con largos períodos de latencia entre la exposición o factor de riesgo y el efecto/enfermedad.

Estudios de Casos y Controles: los grupos de trabajadores que se van a comparar se seleccionan porque presentan o no el efecto o enfermedad ocupacional (E) a estudio. Se elige un grupo de personas que presentan el efecto o enfermedad laboral (E) de interés - casos-, y se comparan, en cuanto a frecuencia de exposición al factor (FR) en estudio, con el grupo que hemos elegido como control (trabajadores igual a los casos, pero sin el efecto o enfermedad ocupacional, por ej. un cáncer ocupacional). Se parte de la identificación del efecto (E) y estudiamos las causas que antecedieron (FR) — carácter retrospectivo del diseño $(\mathbf{E} \Rightarrow \mathbf{F R})$ - a lo largo del tiempo —carácter longitudinal del diseño-. Son estudios longitudinales (varias observaciones), retrospectivos (hacia atrás) y estudian la relación causal del efecto o enfermedad a la causa. El emparejamiento o maching permite una mejor comprensión de los resultados, un equilibrio entre casos y controles y una mejor detección de la interacción entre factores y efectos. En la figura 3 se puede observar la estructura básica de este tipo de estudios.

Figura 3. Estructura básica de un diseño de Casos y Controles

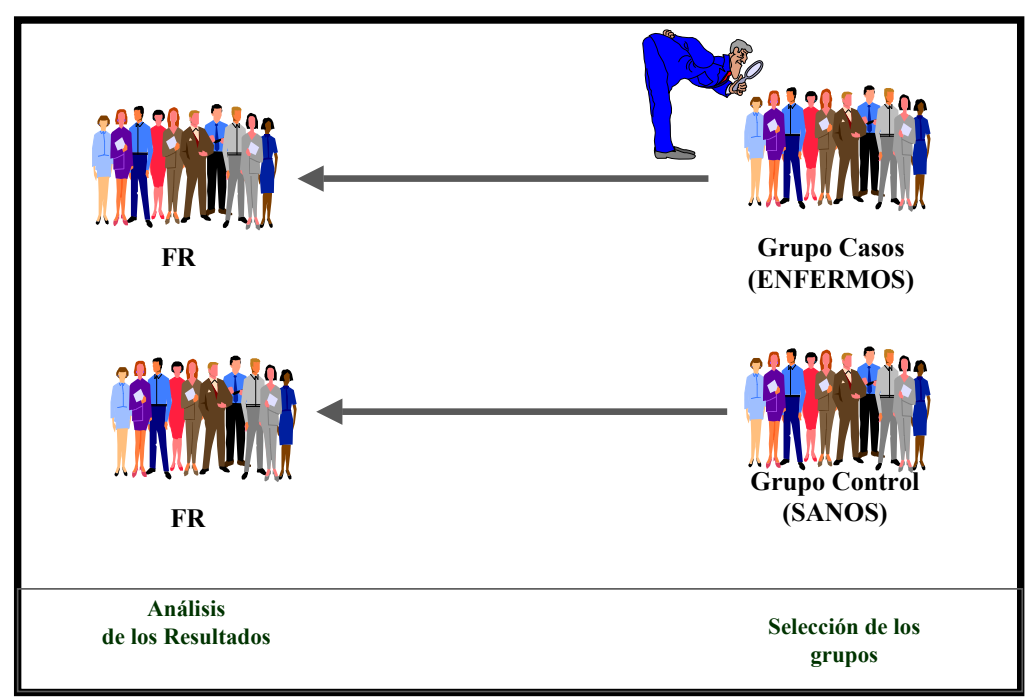


Ventajas: permiten estudiar exposiciones y enfermedades laborales y/o profesionales raras y necesitan poco tiempo, por tanto eficientes.

Inconvenientes: no permiten el cálculo de incidencias, por lo que se utiliza una medida indirecta, la Odds Ratio (OR). El problema es la selección adecuada de los controles y los sesgos. Es el estudio donde se puede cometer un mayor número de sesgos.

\section{DISEÑOS EXPERIMENTALES O DE INTERVENCIÓN}

Cuando tengamos la posibilidad de controlar como investigadores.

\section{Estudios cuasiexperimentales}

Son en parte similares a los experimentales, ya que hay manipulación por parte del investigador en cuanto a la variable exposición de los individuos; la diferencia estriba en que dicha exposición no se estudia de forma individual sino por grupos de población, es decir, no es posible identificar, dentro del grupo expuesto, si todos los individuos, o solamente algunos de ellos, realmente estuvieron bajo el efecto de la variable en estudio. Este tipo de diseños se caracteriza por carecer como mínimo de una de las propiedades de los diseños experimentales: la aleatorización y/o la existencia de grupo control. El objetivo principal de este tipo de estudios es conocer si una intervención o Programa de Salud Laboral está produciendo o no el efecto deseado y diferenciar si los efectos producidos por el Programa son diferentes de los que se hubieran producido de no existir éste, o de haber una intervención alternativa. Pueden ser:

Estudios antes-después: estudio cuasiexperimental que valora la efectividad de una intervención en una empresa o grupo de trabajadores. Puede existir o no grupo control, si existe un grupo similar en el que no se interviene, por ej. con una actividad de Educación Sanitaria en relación con la prevención de una enfermedad profesional. En la figura 4 se puede observar la estructura básica de este tipo de estudios con grupo control.

Figura 4. Estructura básica de un diseño Cuasi-experimental

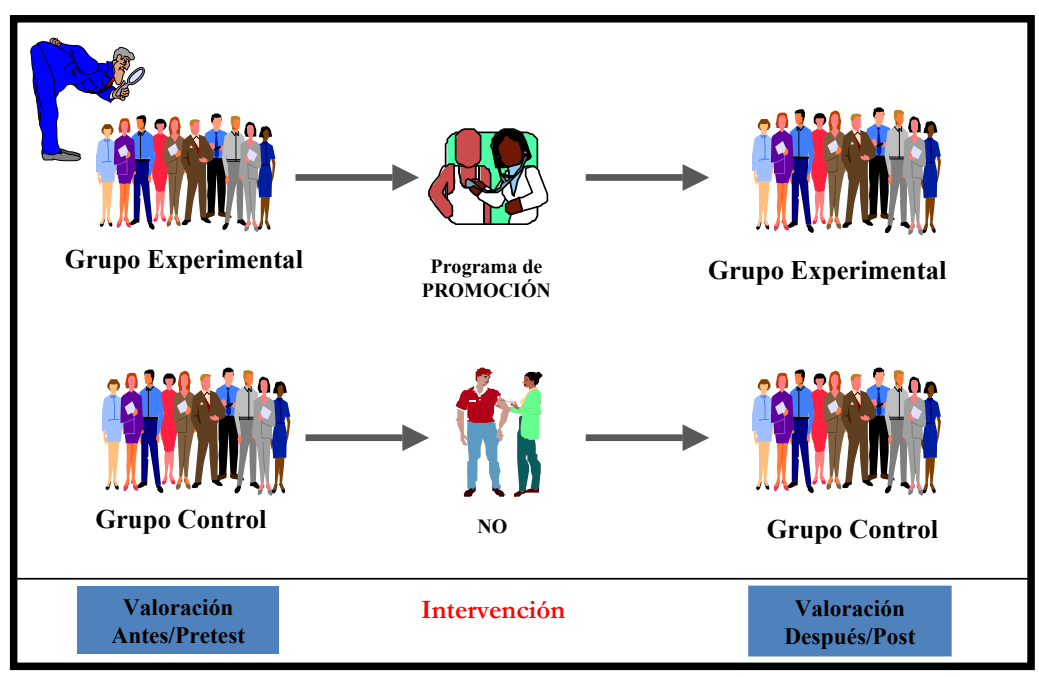

Ensayos de Campo: estudio cuasiexperimental que valora la efectividad de una medida preventiva primaria en una población.

Ensayos Comunitarios: la unidad de asignación es la comunidad, se aplican a individuos sanos y valoran la eficacia de medidas preventivas en poblaciones.

\section{Estudios Experimentales}

Los individuos se estudian a partir de su exposición y el investigador asigna aleatoriamente los sujetos al grupo expuesto - grupo de intervención- y no expuesto -grupo de control- (la asignación de los participantes en los estudios es aleatoria 
individual, es decir, cada participante tiene las mismas posibilidades de pertenecer a un grupo u otro). Son los estudios que ofrecen la mayor validez y rigor en la investigación. Es decir, la mejor evidencia científica.

Ensayos clínicos: estudios aleatorios controlados, valoran nuevos tratamientos - medicamentos - y técnicas diagnósticas en sujetos individuales. Para aumentar la credibilidad de las conclusiones del estudio y evitar sesgos de selección, información y seguimiento se utilizan técnicas de enmascaramiento:

- Simple ciego: el paciente/trabajador desconoce el grupo de tratamiento al que pertenece.

- Doble ciego: el paciente/trabajador como el profesional sanitario desconocen la asignación individual a los grupos de tratamiento, nuevo o placebo.

- Triple ciego: el paciente/trabajador, ni médico, ni evaluador conocen la asignación del tratamiento.

La exposición debe ser presumiblemente beneficiosa para los individuos que la soportarán. Es decir, cumplir el aforismo hipocrático de seguridad del paciente, Primun Non Nocere, ante todo no provocar daño. En relación con éste se ha establecido una legislación específica que arranca con el Código de Nuremberg, regulada en España por la Ley del Medicamento (Ley 25/1990) y el RD 561/1993 sobre requisitos para la realización de ensayos clinicos con medicamentos.

Se trata del diseño epidemiológico metodológicamente más correcto, además, proporciona el más alto nivel de validez de todos los tipos de estudios (prueba más sólida en la que basar inferencias causales y decisiones profesionales). Si además, es multicéntrico, se hace por distintos equipos de investigación en distintos hospitales, ofrece consistencia en los resultados. Los resultados obtenidos son similares por diferentes equipos de investigación en diferentes lugares.

Cada uno de los diseños expuestos tiene ventajas e inconvenientes y a la hora de optar por uno de ellos hemos de partir de la idea de que no hay diseños perfectos, todos pueden conllevar distintos tipos de errores y sesgos. A menudo, además de los factores relacionados con el objetivo del estudio o con el conocimiento previo del tema, factores de orden económico, ético o estratégico y práctico nos harán descartar opciones metodológicamente óptimas pero impracticables, no viables y quedarnos con diseños alternativos que sean viables, factibles y válidos. También puede denominarse Cruzados o Paralelos. En los primeros, a todos los participantes en el ECA se les administra los dos medicamentos, después del primer tratamiento se someten a un período de blanqueo, tiempo para metabolizar y eliminar el fármaco y, después reciben el segundo tratamiento. En los segundos, a cada participante se les administra un solo medicamento o tratamiento. En la figura 5 se puede observar la estructura básica de este tipo de estudios.

Figura 5. Estructura básica de un Ensayo Clínico Aleatorio

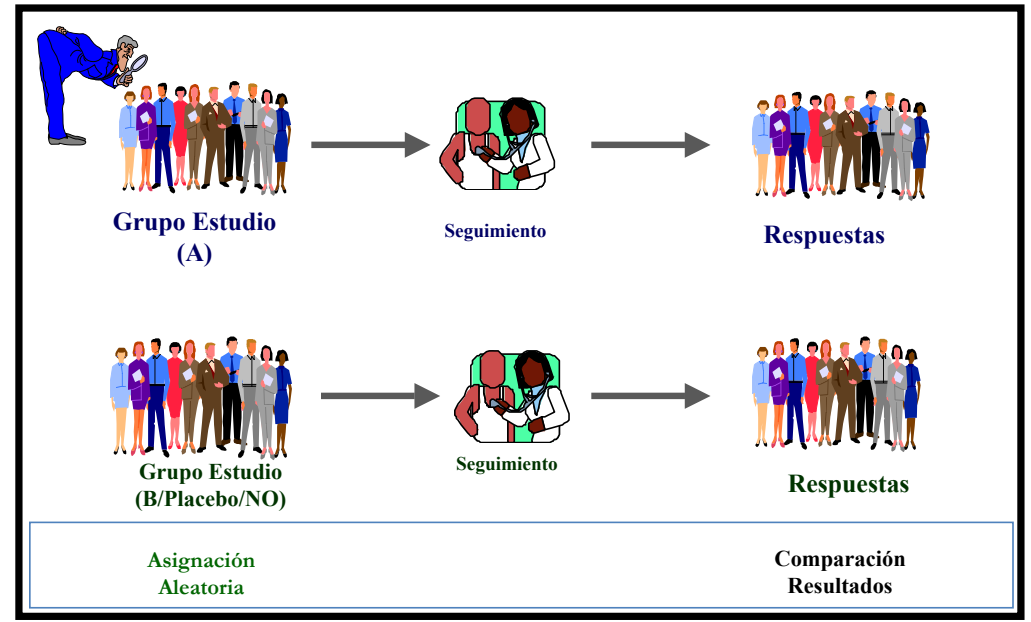


Por último, el proyecto de investigación puede ir encaminado exclusivamente a conocer y sintetizar los conocimientos existentes sobre un tema, bien simplemente con una descripción del estado del tema (Revisión bibliográfica) o bien a través de un metaanálisis de los resultados publicados sobre el mismo.

El meta-análisis consiste en analizar y comparar los resultados de distintos estudios que previamente han sido publicados sobre una misma enfermedad ocupacional y/o profesional independientemente del tipo de diseño elegido en cada caso, para llegar, si es posible, a conclusiones con mayor potencia estadística y metodológica. Aquí, se seleccionan trabajos de investigación, en lugar de pacientes o trabajadores, en base a la pregunta de investigación que se quiere responder y con un mismo tipo de diseño, generalmente, se basan en ensayos clínicos aleatorizados.

Los distintos diseños empleados en investigación, dadas sus características metodológicas, permiten obtener observaciones y resultados con mayor o menor validez y rigor científico, para valorar ésta se han desarrollado distintas escalas, todas ellas están encabezadas por el ensayo clínico aleatorizado multicéntrico. Este trabajo les propone una escala académica fácil de entender y de aplicar en la práctica diaria relacionada con la lectura crítica de artículos científicos y la toma de decisiones (ver figura 6).

Figura 6. Evidencia científica y diseño*

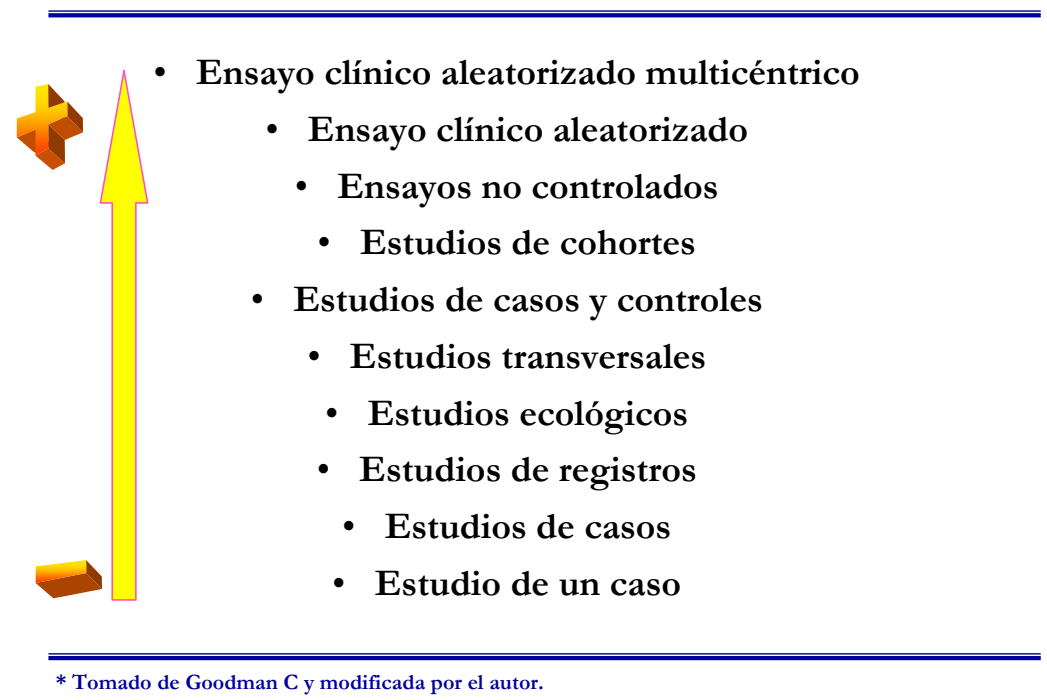

* Tomado de Goodman C y modificada por el autor.

\section{Población de estudio}

Durante la fase de planificación es importante seleccionar la población que se quiere investigar (población de estudio). Los sujetos de observación pueden ser: individuos aislados o comunidades (urbanas o rurales), instituciones (hospitalarias o de otra índole), trabajadores, empresas, ámbitos y sectores laborales, etc.

Desde un principio debe quedar claro y explicitado lo que se va a considerar como unidad de análisis (trabajadores, incidentes, accidentes, seguimiento, sector laboral, ámbito, etc.) y las características imprescindibles que deben cumplir éstas para formar parte de la población a investigar.

Las decisiones sobre este tipo de criterios vienen determinadas por el interés en conformar una población más homogénea, o por el propio problema a investigar. La decisión sobre estos criterios conlleva, sin embargo, una limitación a la hora de extender los resultados a otras poblaciones — validez externa - , ya que éstas sólo serán aplicables a los sujetos que cumplan los mismos criterios utilizados en nuestro estudio.

Es necesario, por tanto, tener en mente el elegir sólo aquellos criterios de inclusión que verdaderamente den mayor significación clínica y estadística al estudio, porque a 
medida que éstos aumentan, disminuyen las posibles inferencias y generalizaciones de los resultados obtenidos.

El esfuerzo por parte de los investigadores irá encaminado a dejar constancia, escrita a ser posible, de cada uno de estos criterios, especificando los procedimientos y medidas utilizadas para determinar la elegibilidad de los sujetos. La realización correcta de este paso es imprescindible para conseguir una adecuada validez interna del estudio.

\section{MUESTREO}

Una vez deciddidas las características de los trabajadores que formarán parte de la población sometida a estudio, ha de establecerse el número de trabajadores que es necesario incluir en el mismo. Un estudio se puede realizar:

- Sobre todos los sujetos disponibles de un sector laboral o empresa, entonces se hará en el universo de sujetos, o sea, un estudio poblacional.

- Sobre una muestra representativa de la población de referencia o diana, con un tamaño lo suficientemente amplio para permitir el conocimiento del evento, es decir, habrá un tamaño de muestra mínimo para garantizar el buen estudio del evento (el tamaño de muestra así estipulado será fijo).

Cuando queremos investigar un fenómeno en una determinada población no es necesario estudiar a todos y cada uno de los trabajadores de la misma, sino que analizando una muestra representativa, correctamente extraída de ese grupo de trabajadores, podremos transportar - inferir - nuestras conclusiones al conjunto de la población a estudio.

El proceso de selección debe ser el azar, de modo que cada uno de los trabajadores de la empresa objeto de estudio tenga la misma probabilidad que los demás de ser seleccionado, sin desviación a favor de algún subgrupo. Para obtener una muestra aleatoria puede usarse una tabla de números aleatorios (disponibles en los manuales de introducción a la estadística). Otras formas frecuentes de obtener muestras sistemáticas consisten en tomar consecutivamente una de cada tres personas de una lista o aquellas cuyo número de orden acabe en 0 o en 5.

Un proceso inductivo de esta naturaleza se asocia inevitablemente a la posibilidad de cometer errores, mayores cuanto menor sea el tamaño de la muestra y/o menor su representatividad para la realidad que estamos estudiando. Dicho error representa la probabilidad de que los resultados obtenidos en la muestra no se correspondan con lo que ocurriría si trabajáramos con toda la población a estudio.

Obtener una buena muestra significa obtener una versión significativa de la población que reproduzca sus rasgos básicos. Dos condiciones son inherentes a la extracción estadísticamente representativa de una muestra:

- Que el método otorgue una probabilidad conocida de integrar en la muestra a cada unidad de la población.

- Que tal probabilidad no sea nula para elemento alguno.

Recordar que cuando se extrae una muestra de una población para estudiarla, no nos interesan sólo las personas de la muestra, lo que queremos es usar la muestra para saber algo determinado de la población de donde procede.

El tamaño de la muestra depende directamente de la incidencia que el fenómeno de interés presenta en la población que se quiere estudiar, es decir, a partir de cuándo se atribuirá la frecuencia de aparición de un efecto determinado al factor en estudio y no al azar.

El conocer la incidencia o la prevalencia de un fenómeno depende del conocimiento previo del tema de estudio, y en el caso de que éste no exista, lo apropiado es pensar en 
la opción más desfavorable, es decir, que exista igual probabilidad de que un sujeto presente el efecto de interés como de que no lo presente.

El tamaño de la muestra depende además de consideraciones del trabajo, de las variables que se van analizar y de los cruces a presentar, del nivel de medición de las variables y de los diferentes grupos a comparar (a mayor complejidad, mayor será el tamaño de la muestra). Los cálculos estadísticos para conocer el número definitivo de sujetos a estudiar son complicados y diferentes según el tipo de estudio que se haya elegido.

Según el tipo de estudio y la finalidad de la misma, la muestra puede ser:

- Representativa: teniendo las mismas características del universo.

- Seleccionada: escogiendo una categoría de interés en la población de estudio para revisar un aspecto especial.

\section{FUENTES DE INFORMACIÓN DE LOS SUJETOS}

La fuente de los sujetos es importante para la inferencia. Se debe tener en consideración la variabilidad y las características de los sujetos según persona, tiempo y lugar. También se debe ver la relación de la población de estudio con la población de referencia correspondiente y la población general.

Igualmente se debe tener presente en la selección de los individuos que van a participar en el estudio la distribución en grupos de acuerdo con la exigencia metodológica.

Como regla general es conveniente hacer siempre una revisión de las fuentes de datos e información disponibles, antes de optar por diseñar e implantar un registro nuevo que suponga cargas de trabajo adicionales y a menudo suplementarias con las ya existentes.

Son muchas las posibilidades de obtención de datos, y sólo cuando éstos no estén previamente disponibles, o cuando las dudas sobre la fiabilidad de los mismos sean razonables, se procederá a diseñar un cuestionario o registro nuevo.

\section{ANÁLISIS}

La valoración es una fase que todo tipo de estudio tiene que tener, que no debe olvidarse, pues forma parte del proceso de investigación y dependiente de todo lo realizado. Los datos e información obtenida de un estudio mal diseñado, difícilmente se van a mejorar en el análisis como para proporcionar unas conclusiones válidas y consistentes. El análisis por muy exhaustivo y riguroso que sea no va mejorar la calidad, pertinencia y validez de los datos. No es, por tanto, una herramienta o varita mágica que mejore la vulnerabilidad del diseño y de la metodología.

Al terminar la realización de un estudio epidemiológico, las conclusiones se refieren en primera instancia a la población de estudio sobre la cual se hace el ensayo. Se debe tratar de ampliar o inferir las conclusiones a otra población mayor, que es la población de riesgo, y aún más, a la población de referencia (aquella que además de incluir a la población de riesgo actual, representa a la población pasada, presente y futura).

La interpretación de los datos para su ampliación a la población de referencia -inferencia científica-, comprende la interpretación del análisis estadístico, el establecimiento de las bases para causalidad e inferencia, el planteamiento de la validez de las conclusiones, los alcances y limitaciones de las conclusiones y de la inferencia.

Se hace en base a: Los conocimientos anteriores existentes. 
La metodología empleada en la investigación.

Los resultados obtenidos en la investigación.

Es de suma importancia realizar un buen análisis y una interpretación correcta de los hallazgos para que sean válidos y poder ampliar o inferir las conclusiones a una población mucho mayor que la que participó directamente en el estudio. La presencia de un error puede falsear las conclusiones o la inferencia final.

En los Anexos se presentan los análisis epidemiológicos de los estudios de cohortes y casos y controles.

\section{BIBLIOGRAFÍA}

1. Aibar Remón C, Rabaneque MJ, Álvarez-Dardet C, Nolasco A, Moncho J, Gascón E. Evolución de los diseños epidemiológicos de la investigación clínica en España. Rev Esp Salud Púb. 1999; 73:445-453.

2. Argimón Pallás JM, Jiménez Villa J. Métodos de investigación clínica y epidemiológica. Barcelona: Elservier. 2006.

3. Baum F. Investigación en Salud Pública: el debate entre las metodologías cuantitativas y cualitativas. Rev de Salud Pública. 1997; 5:175-193.

4. Burgos Rodríguez R, et al. Metodología de investigación y escritura científica en clínica. Granada: Escuela Andaluza de Salud Pública. 1998.

5. Caycoya M, Mirón Canelo JA. Cáncer de pulmón en Asturias. Un estudio de casos y controles. Gac Sanit (Barc). 2003; 17:226-230.

6. García Criado EI. Investigación en atención primaria. Rev Semergen. 2001; 27:190-211.

7. Garrido Cantarero G, et al. Golberg E, Conde J, De la Cal MA, Martín Moreno JM. Guía para la evaluación de proyectos de investigación en Ciencias de la Salud. Med Clin (Barc). 1977; 108:460-471.

8. Kleinbaum DG, Kupper LL, MorgensternH. Epidemiologic research. Priciples and quantitative methods. Belmont, CA Lifetime Learning Publications. 1982.

9. Mirón Canelo JA, Pino Montes J, Arroyo V, Sáenz González MC. Epidemiological study of Paget's disease of bone in a zone of the Province of Salamanca (Spain). The Paget's disease of the bone study group of Salamanca. European Journal Epidemiology. 1997; 13:801-805.

10. Mirón Canelo JA, Sáenz González MC. Efectividad de un seminario informativo sobre Salud laboral. Aten Primaria. 1999; 23:467-473.

11. Mirón Canelo JA, Alonso Sardón M. Medidas de frecuencia, asociación e impacto. Rev Medicina y Seguridad en el Trabajo. 2008; 54:93-102.

12. Rebagliato M, Ruiz I, Arranz M. Metodología de investigación en Epidemiología. Madrid: Diaz de Santos. 1996.

13. Sackett DL. Medicina Basada en la evidencia. Como ejercer y enseñar la Medicina Basada en la Evidencia. Madrid: Churchil Livingstone. 1997.

14. Salleras Sanmartí L. La investigación en poblaciones humanas. Med Clin (Barc). 1989; 92:264-268.

15. Sanz-Valero J, Veiga-Cabo J, Rojo-Alonso C, D’Agostino M, Wanden-Berghe C, Xavier Espulgues Pellicer J, Rodrigues Guilam C. Los filtros metodológicos: aplicación a la búsqueda bibliográfica en la medicina del trabajo española. Med Segur Trab. 2008; 54(211):75-83.

16. Veiga de Cabo J, Fuente Díaz E, Zimmermann Verdejo M. Modelos de estudios en investigación aplicada: conceptos y criterios para el diseño. Med Segur Trab. [online]. 2008; 54(210):81-88.

17. Villar Álvarez F, Banegas Banegas JR, González Enriquez J, Martín Moreno JM, Rodríguez Artalejo F. Diseño y análisis epidemiológico. Rev ROL de Enfermería. 1987; 112:29-32. 


\section{ANEXOS}

SEGUIMIENTO EN UN ESTUDIO DE COHORTES

1.- Construir la tabla de contingencia:

\begin{tabular}{|c|c|c|c|}
\hline & $\mathbf{E}$ & $\mathbf{S}$ & \\
\hline $\mathbf{F R}+$ & $\mathrm{a}$ & $\mathrm{b}$ & $\mathbf{M}_{1}$ \\
\hline $\mathbf{F R}-$ & $\mathrm{c}$ & $\mathrm{d}$ & $\mathbf{M}_{2}$ \\
\hline & $\mathbf{N}_{1}$ & $\mathbf{N}_{2}$ & $\mathbf{N}$ \\
\hline
\end{tabular}

$$
\begin{array}{ll}
\mathrm{M}_{1}=\text { EXPUESTOS } & \mathrm{N}_{1}=\text { ENFERMOS } \\
\mathrm{M}_{2}=\text { NO EXPUESTOS } & \mathrm{N}_{2}=\text { SANOS }
\end{array}
$$

\section{2.- HIPÓTESIS: $\quad I_{e}>I_{o}$; es decir, $\quad a / M_{1}>c / M_{2}$.}

3.- ASOCIACIÓN: ¿Existe significación estadística entre el FR y E?.

$$
\mathbf{X}_{\text {MANTEl-hatnszel }}^{2}=\frac{(a * d-b * c)^{2}}{M_{1} * M_{2} * N_{1} * N_{2}} \times(\mathrm{N}-1)
$$

$\mathrm{X}_{\text {calculado }}^{2}<\mathrm{X}_{\text {teórico }}^{2} \Rightarrow \mathrm{NO}$ existe asociación estadística significativa entre FR y E

$\mathrm{X}_{\text {calculado }}^{2}>\mathrm{X}_{\text {teórico }}^{2} \Rightarrow \mathrm{SI}$ existe asociación estadística significativa entre FR y E

Este test de significancia se traduce en términos de probabilidad (p) para un nivel de confianza

$$
0.95 \rightarrow \mathrm{p}<0.05 \Rightarrow \mathrm{X}_{\text {teórico }}^{2}=3.84
$$

4.- CAUSALIDAD: ¿Existe asociación causal entre el FR y E?.

- Para medir la magnitud o fuerza de asociación: RIESGO RELATIVO (RR)

$$
\mathrm{RR}=\frac{I e}{I o}
$$

$\mathrm{RR}<1 \Rightarrow$ el FR protege de la aparición de $\mathrm{E}$

$\mathrm{RR}=1 \Rightarrow$ no existe relación entre FR y $\mathrm{E}$

$\mathrm{RR}>1 \Rightarrow$ el FR influye en la aparición de $\mathrm{E}$

- Para inferir causalidad -precisión de la magnitud-: INTERVALO DE CONFIANZA

$$
\mathrm{IC} \text { del RR }=\mathrm{RR}^{\left(1 \pm \frac{Z \alpha}{\sqrt{X^{2}}}\right)}
$$

donde $Z$ para un $\alpha=p=0.05 \Rightarrow Z \alpha=1.96$ cuanto más pequeño el intervalo mayor la precisión del estudio si la asociación es significativa nunca incluirá el valor 1 


\section{SEGUIMIENTO EN UN ESTUDIO DE CASOS y CONTROLES}

1.- Construir la tabla de contingencia:

\begin{tabular}{|c|c|c|c|}
\hline & $\mathbf{E}$ & $\mathbf{S}$ & \\
\hline $\mathbf{F R}+$ & $\mathrm{a}$ & $\mathrm{b}$ & $\mathbf{M}_{1}$ \\
\hline $\mathbf{F R}-$ & $\mathrm{c}$ & $\mathrm{d}$ & $\mathbf{M}_{2}$ \\
\hline & $\mathbf{N}_{1}$ & $\mathbf{N}_{2}$ & $\mathbf{N}$ \\
\hline
\end{tabular}

$\mathrm{M}_{1}=$ EXPUESTOS

$\mathrm{N}_{1}=$ CASOS

$\mathrm{M}_{2}=$ NO EXPUESTOS

$\mathrm{N}_{2}=$ CONTROLES

2.- HIPÓTESIS: $\quad \mathrm{p}_{1}>\mathrm{p}_{2}$; es decir, $\quad \mathrm{a} / \mathbf{N}_{1}>\mathrm{b} / \mathbf{N}_{2}$.

3.- ASOCIACIÓN: ¿Existe significación estadística entre el FR y E?

$$
\mathbf{X}_{\text {MANTEL-HAENSZEL }}^{2}=\frac{(a * d-b * c)^{2}}{M_{1} * M_{2} * N_{1} * N_{2}} \times(\mathrm{N}-1)
$$

$\mathrm{X}_{\text {calculado }}^{2}<\mathrm{X}_{\text {térico }}^{2} \Rightarrow \mathrm{NO}$ existe asociación estadística significativa entre FR y E

$\mathrm{X}_{\text {calculado }}^{2}>\mathrm{X}_{\text {teórico }}^{2} \Rightarrow \mathrm{SI}$ existe asociación estadística significativa entre FR y E

Este test de significancia se traduce en términos de probabilidad (p) para un nivel de confianza

$$
0.95 \rightarrow \mathrm{p}<0.05 \Rightarrow \mathrm{X}_{\text {térico }}^{2}=3.84
$$

4.- CAUSALIDAD: ¿Existe asociación causal entre el FR y E?

- Para medir la magnitud o fuerza de asociación: ODDS RATIO (OR).

$$
\mathrm{OR}=\frac{a^{*} d}{b^{*} c}
$$

$\mathrm{OR}<1 \Rightarrow$ el FR protege de la aparición de $\mathrm{E}$

$\mathrm{OR}=1 \Rightarrow$ no existe relación entre FR y E

$\mathrm{OR}>1 \Rightarrow$ el FR influye en la aparición de $\mathrm{E}$

- Para inferir causalidad -precisión de la magnitud-: INTERVALO DE CONFIANZA

$$
\mathrm{IC} \text { del OR }=\mathrm{OR}^{\left(1 \pm \frac{Z \alpha}{\sqrt{X^{2}}}\right)}
$$

donde $Z$ para un $\alpha=p=0.05 \Rightarrow Z \alpha=1.96$

cuanto más pequeño el intervalo mayor la precisión del estudio

si la asociación es significativa nunca incluirá el valor 1

\section{5.- FRACCIÓN ETIOLÓGICA: valorar el impacto.}

$$
\mathrm{FE}_{\mathrm{e}}=\frac{O R-1}{O R} \times 100 \quad \mathrm{FE}_{\mathrm{p}}=\mathrm{P}_{\mathrm{c}} \times \mathrm{FE}_{\mathrm{e}} \Rightarrow \text { donde } \mathrm{P}_{\mathrm{c}}=\mathrm{a} / \mathrm{N}_{1}
$$

